This document is the accepted manuscript version of the following article:

Kophimai, Y., Cornejo, C., Peintinger, M., Bergamini, A., \& Scheidegger, C. (2011). Characterization of nuclear microsatellite loci in the calcareous fen specialist Scorpidium Cossonii (Cal1iergonaceae). American Journal of Botany, 98(10), e290-e292. https://doi.org/10.3732/ajb.1100144

AJB Primer Notes \& Protocols-"Scorpidium microsatellites" Kophimai et al. - 1

\title{
Characterization of nuclear microsatellite loci in the calcareous fen
}

\section{specialist Scorpidium cossonii (Calliergonaceae) ${ }^{1}$}

Yuppayao Kophimai ${ }^{2,4}$, Carolina Cornejo ${ }^{2}$, Markus Peintinger ${ }^{3}$, Ariel Bergamini $^{2}$ and Christoph Scheidegger ${ }^{2}$

\author{
${ }^{2}$ WSL Swiss Federal Research Institute, Zürcherstrasse 111, CH-8903 Birmensdorf, \\ Switzerland \\ ${ }^{3}$ Institute of Evolutionary Biology and Environmental Studies, University of Zurich, \\ Winterthurerstrasse 190, CH-8057 Zürich, Switzerland
}

Email addresses: YK: yuppayao.kophimai@wsl.ch

CC: carolina.cornejo@wsl.ch

MP: peinti@t-online.de

AB: ariel.bergamini@wsl.ch

CS: christoph.scheidegger@wsl.ch

Number of words: 961

${ }^{1}$ Manuscript received ; revision accepted

${ }^{4}$ Author for correspondence: yuppayao.kophimai@wsl.ch

The authors thank the Genetic Diversity Centre, ETH Zurich, for technical assistance and S. Werth for comments on a previous version of the manuscript. This study was supported by a scholarship to YK (Higher Educational Strategic Scholarships for Frontier Research Network) and grants from the Federal Office for the Environment (FOEN) to CS (MfM-U Biodiversity) and AB (06.0126.PZ / I244-0978). 
AJB Primer Notes \& Protocols-"Scorpidium microsatellites"

Kophimai et al. - 2

\section{ABSTRACT}

- Premise of the study: Nuclear microsatellite markers were developed in the fen specialist moss, Scorpidium cossonii (Schimp.) Hedenäs, to study genetic diversity and genetic structure of this species in relation to land-use types, habitat fragmentation and habitat conservation measures.

- Methods and Results: Fourteen polymorphic microsatellite markers were isolated and characterized using an enrichment method. All primers were tested on 140 gametophytes collected from four populations in Switzerland. The primers amplified di- and trinucleotide repeats with three to 26 alleles per locus. The primers worked also in the two other species of the genus: All and twelve primers successfully amplified in S. revolvens and S. scorpioides, respectively.

- Conclusions: In future studies, our primers will provide valuable information on genetic diversity, genetic structure as well as on historical and recent gene flow in $S$. cossonii and they enable related research in other Scorpidium species.

Key words: bryophytes; calcareous fens; cross-species amplification; microsatellites; 


\section{METHODS AND RESULTS}

We pooled DNA of $S$. cossonii specimens sampled from six different populations in Northern Switzerland. Ecogenics GmbH (Zurich, Switzerland) developed a genetic library from size selected genomic DNA ligated into SNX forward/SNX reverse linker (Hamilton et al., 1999) and enriched by magnetic bead selection with biotin-labelled $(\mathrm{CT})_{13},(\mathrm{GT})_{13}$, $(\mathrm{AAC})_{10}$ and (ATC) $)_{10}$ oligonucleotide repeats (Gautschi et al., 2000a,b). Of 528 recombinant 
colonies screened, 341 gave a positive signal after hybridization. Plasmids from 48 positive clones were sequenced and primers were designed for 20 microsatellite inserts, of which 14 were tested for polymorphism.

The microsatellite loci were tested for variability on 140 gametophytes of $S$. cossonii collected from four populations in the pre-Alps of north-eastern Switzerland. Total genomic DNA was extracted from stem tissue using the DNeasy 96 plant kit (Qiagen, Hombrechtikon, Switzerland). All forward primers were labelled with one fluorescent dye at the 5 ' end region and three PCR multiplexes were set up (Table 1). PCR reactions were performed in a volume of $5 \mu$ l containing about 1-5 ng genomic DNA, varying primer concentrations (Table 1) and 1x multiplex PCR master mix (Qiagen). PCR cycles were started with 15 min at $95^{\circ} \mathrm{C}$ initial denaturation, followed by 30 cycles of $30 \mathrm{~s}$ at $94^{\circ} \mathrm{C}, 90 \mathrm{~s}$ at annealing temperature (Table1), $1 \mathrm{~min}$ at $72^{\circ} \mathrm{C}$, ending with a final extension step of $60 \mathrm{~min}$ at $60^{\circ} \mathrm{C}$. PCR products were run on a 3130xl Genetic Analyser (Applied Biosystems, Foster City, California, USA) using LIZ500 as internal size standard and alleles were sized with GENEMAPPER 3.7 (Applied Biosystems). The variability of each microsatellite locus was measured by counting the number of alleles and calculating gene diversity using Arlequin version 3.11 (Excoffier et al., 2005).

Among the 14 microsatellite motifs, nine were dinucleotide repeats; three were trinucleotide repeats and two were combinations between two different trinucleotide repeats (Table 1). The microsatellite loci produced three to 26 alleles per locus and mean gene diversities over four populations varied from 0.13 to 0.68 (Table 2). Three individuals of each of two closely related species (Hedenäs et al., 2009), S. revolvens (Sw. ex anon.) Rubers and S. scorpioides (Hedw.) Limpr. were tested for cross-species amplifications. After PCR 
optimization for the annealing temperature, all 14 primers successfully amplified in $S$. revolvens and 12 primers (except Sc2 and Sc22) in S. scorpioides.

\section{CONCLUSIONS}

The polymorphic microsatellite loci reported here are a promising tool for investigations of current genetic diversity, genetic structure and historical as well as recent gene flow in S. cossonii. This research will considerably improve our basic understanding of the genetic structure of fragmented bryophyte populations and it will serve as a base for the development of conservation strategies in this highly specialised fen species. Furthermore, because these loci are applicable to other Scorpidium species, they can be widely used and a number of possible studies using these microstellites are in progress.

\section{LITERATURE CITED}

Bergamini, A., M. Peintinger, S. FAKHERAn, H. Moradi, B. SCHMid, AND J. Joshi. 2009. Loss of habitat specialists despite conservation management in fen remnants 19952006. Perspectives in Plant Ecology Evolution and Systematics 11: 65-79.

EXCOFFIER, L., G. LAVAL, AND S. SCHNEIDER. 2005. Arlequin ver. 3.0: An integrated software package for population genetics data analysis. Evolutionary Bioinformatics Online 1: $47-50$.

GAUTSCHI, B., A. WIDMER, AND J. KOELLA. 2000a. Isolation and characterization of microsatellite loci in the dice snake (Natrix tessellata). Molecular Ecology 9: 21912193.

GAUTSCHI, B., I. TENZER, J. P. MÜLLER, AND B. SCHMID. 2000b. Isolation and characterization of microsatellite loci in the bearded vulture (Gypaetus barbatus) and crossamplification in three Old World vulture species. Molecular Ecology 9: 2193-2195. 
AJB Primer Notes \& Protocols-"Scorpidium microsatellites"

Kophimai et al. -6

HAJKOVA, P., M. HAJEK, AND K. KinTROVA. 2009. How can we effectively restore species richness and natural composition of a Molinia-invaded fen? Journal of Applied Ecology 46: 417-425.

Hamilton, M. B., E. L. Pincus, A. Di Fiore, AND R. C. FleisCheR. 1999. Universal linker and ligation procedures for construction of genomic DNA libraries enriched for microsatellites. Biotechniques 27: 500-507.

HEDENÄS L. 1989. The genera Scorpidium and Hamatocaulis, gen. nov., in northern Europe. Lindbergia 15: 8-36.

HEDENÄS L. 2009. Relationships among artic and non-arctic haplotypes of the moss species Scorpidium cossonii and Scorpidium scorpioides (Calliergonaceae). Plant Systematics and Evolution 277: 217-231.

LIENERT, J., M. FISCHER, J. SCHNELLER, AND M. DIEMER. 2002. Isozyme variability of the wetland specialist Swertia perennis (Gentianaceae) in relation to habitat size, isolation, and plant fitness. American Journal of Botany 89: 801-811.

Peintinger, M., A. Bergamini, AND B. SCHMID. 2003. Species-area relationships and nestedness of four taxonomic groups in fragmented wetlands. Basic and Applied Ecology 4: 385-394. 
AJB Primer Notes \& Protocols-"Scorpidium microsatellites"

Kophimai et al. -7

TABLE 1. Characteristics of 14 nuclear microsatellite loci in S. cossonii. For each locus, the forward and reverse primer sequences, repeat motif, labelled fluorescent dye, size of the original fragment, PCR multiplex group, primer concentration, annealing temperature when run in multiplex (Ta) and GenBank accession number are shown.

\begin{tabular}{|c|c|c|c|c|c|c|c|c|}
\hline Locus & $\begin{array}{l}\text { Primer sequence } \\
\text { forward/reverse }\end{array}$ & Repeat motif & $\begin{array}{l}\text { Fluorescent } \\
\text { dye }\end{array}$ & $\begin{array}{l}\text { Size } \\
\text { (bp) }\end{array}$ & Multiplex & $\begin{array}{l}\text { Primer conc. } \\
(\mu \mathrm{M})\end{array}$ & $\begin{array}{l}\mathrm{Ta} \\
\left({ }^{\circ} \mathrm{C}\right)\end{array}$ & $\begin{array}{l}\text { GenBank } \\
\text { accession no. }\end{array}$ \\
\hline \multirow[t]{2}{*}{$\mathrm{Sc} 01$} & AGCCAAAACGTATGGAAACC & $(\mathrm{AG})_{24}$ & FAM & 178 & 1 & 0.14 & 59 & JF700218 \\
\hline & GATAGGAAGGCAGTGCAACC & & & & & 0.14 & & \\
\hline \multirow[t]{2}{*}{$\mathrm{Sc} 02$} & AAATGGAGTATTGGATGAGATGG & $(\mathrm{AC})_{13}$ & NED & 159 & 1 & 0.21 & 59 & JF700219 \\
\hline & CTAATGCAAGGGCATGAGTG & & & & & 0.21 & & \\
\hline \multirow[t]{2}{*}{$\mathrm{Sc} 03$} & CTCAAGTCACGGCTGCTTC & $(\mathrm{GT})_{16}$ & NED & 149 & 2 & 0.08 & 59 & JF700220 \\
\hline & CAAAGGGACTTCAATTCCAAC & & & & & 0.08 & & \\
\hline \multirow[t]{2}{*}{$\mathrm{Sc} 04$} & TGTGAACGTGGAAGCTTGTG & $(\mathrm{GT})_{17}$ & PET & 137 & 1 & 0.07 & 59 & JF700221 \\
\hline & GCCCGAAGTAGTTGCTTCTC & & & & & 0.07 & & \\
\hline \multirow[t]{2}{*}{$\mathrm{Sc} 07$} & AGGATGAGAGTTCCCTGGTG & $(\mathrm{CA})_{13}$ & FAM & 205 & 3 & 0.08 & 60 & JF700222 \\
\hline & TGGCATTCAAACACACATCC & & & & & 0.08 & & \\
\hline \multirow[t]{2}{*}{$\mathrm{Sc} 09$} & ACGTCAAGTGACCCCACAC & $(\mathrm{AC})_{22}$ & VIC & 156 & 1 & 0.07 & 59 & JF700223 \\
\hline & TTGGGTAGTTTGGCGTTAGG & & & & & 0.07 & & \\
\hline \multirow[t]{2}{*}{ Sc13 } & ACAGCAGCACATGTCCAGAG & $(\mathrm{CAA})_{9}$ & PET & 143 & 2 & 0.08 & 59 & JF700224 \\
\hline & GAGGTAATCCAGCAGCCATC & & & & & 0.08 & & \\
\hline \multirow[t]{2}{*}{$\operatorname{Sc} 16$} & AATCAAGGCCCCATCTAAGC & $(\mathrm{CAT})_{13}(\mathrm{CAA})_{4}$ & FAM & 208 & 2 & 0.08 & 59 & JF700225 \\
\hline & TCACAGATCGCAAACAAACC & & & & & 0.08 & & \\
\hline \multirow[t]{2}{*}{$\mathrm{Sc} 17$} & GTGTGAACCTGCACGAGATG & $(\mathrm{GTT})_{8}(\mathrm{GCT})_{6}$ & VIC & 191 & 2 & 0.08 & 59 & JF700226 \\
\hline & CATGGCCGTAAGAGTGGAAC & & & & & 0.08 & & \\
\hline \multirow[t]{2}{*}{$\mathrm{Sc} 18$} & CTTCAGAAAGCCGATCAAGC & $(\mathrm{CAA})_{8}$ & NED & 127 & 3 & 0.08 & 60 & JF700227 \\
\hline & TTGCGGAAGATCTCCTCTTG & & & & & 0.08 & & \\
\hline \multirow[t]{2}{*}{ Sc19 } & GCGCTTGTATAAGCCTGAGC & $(\mathrm{GTT})_{7}$ & PET & 135 & 3 & 0.08 & 60 & JF700228 \\
\hline & TGCCACTAACGAAGGGACTG & & & & & 0.08 & & \\
\hline \multirow[t]{2}{*}{ Sc20 } & CGCTCTAATTGAGGCATTTG & $(\mathrm{AC})_{20}$ & VIC & 172 & 3 & 0.16 & 60 & JF700229 \\
\hline & TGGTTTCGATTTCGGACAAG & & & & & 0.16 & & \\
\hline \multirow[t]{2}{*}{$\mathrm{Sc} 21$} & TGGAGGCTTGTCCAGAATTG & $(\mathrm{TG})_{13}$ & FAM & 93 & 3 & 0.08 & 60 & JF700230 \\
\hline & CGACACTGCAAACCAACTAGAC & & & & & 0.08 & & \\
\hline \multirow[t]{2}{*}{$\mathrm{Sc} 22$} & GGCATCCCTACCTCATTGACCG & $(\mathrm{CA})_{22}$ & FAM & 159 & 2 & 0.16 & 59 & JF700231 \\
\hline & GGGGGCTTTCACCAGGAATTCA & & & & & 0.16 & & \\
\hline
\end{tabular}


TABLE 2. Results of the initial primer screening in four populations of S. cossonii. Shown for each primer are the number of alleles observed $\left(\mathrm{N}_{\mathrm{A}}\right)$ and gene diversity $(\mathrm{He})$. Sample sizes per population and geographical coordinates (Swiss grid) are given in parentheses.

\begin{tabular}{|c|c|c|c|c|c|c|c|c|c|c|}
\hline \multirow[b]{2}{*}{ Locus } & \multicolumn{2}{|c|}{$\begin{array}{l}\text { Population } 1(\mathrm{~N}=35) \\
(725.83 \mathrm{E}, 234.95 \mathrm{~N})\end{array}$} & \multicolumn{2}{|c|}{$\begin{array}{l}\text { Population } 2(\mathrm{~N}=35) \\
(703.45 \mathrm{E}, 222.48 \mathrm{~N})\end{array}$} & \multicolumn{2}{|c|}{$\begin{array}{l}\text { Population } 3(\mathrm{~N}=35) \\
(712.21 \mathrm{E}, 220.75 \mathrm{~N})\end{array}$} & \multicolumn{2}{|c|}{$\begin{array}{l}\text { Population } 4(\mathrm{~N}=35) \\
(707.16 \mathrm{E}, 220.40 \mathrm{~N})\end{array}$} & \multirow{2}{*}{$\begin{array}{l}\text { Total } \\
\mathrm{N}_{\mathrm{A}}\end{array}$} & \multirow{2}{*}{$\begin{array}{c}\text { Mean } \\
\mathrm{He}\end{array}$} \\
\hline & $\mathrm{N}_{\mathrm{A}}$ & $\mathrm{He}$ & $\mathrm{N}_{\mathrm{A}}$ & $\mathrm{He}$ & $\mathrm{N}_{\mathrm{A}}$ & $\mathrm{He}$ & $\mathrm{N}_{\mathrm{A}}$ & $\mathrm{He}$ & & \\
\hline $\mathrm{Sc} 01$ & 9 & 0.82 & 11 & 0.89 & 7 & 0.31 & 6 & 0.70 & 26 & 0.68 \\
\hline $\mathrm{Sc} 02$ & 4 & 0.66 & 6 & 0.58 & 4 & 0.31 & 7 & 0.67 & 13 & 0.55 \\
\hline $\mathrm{Sc} 03$ & 3 & 0.51 & 3 & 0.54 & 3 & 0.26 & 7 & 0.70 & 8 & 0.50 \\
\hline $\mathrm{Sc} 04$ & 4 & 0.66 & 4 & 0.57 & 6 & 0.35 & 3 & 0.54 & 9 & 0.53 \\
\hline $\mathrm{Sc} 07$ & 3 & 0.64 & 4 & 0.39 & 2 & 0.05 & 5 & 0.62 & 8 & 0.42 \\
\hline $\mathrm{Sc} 09$ & 4 & 0.66 & 7 & 0.65 & 5 & 0.31 & 7 & 0.70 & 13 & 0.58 \\
\hline Sc13 & 2 & 0.44 & 1 & 0.00 & 2 & 0.05 & 2 & 0.05 & 3 & 0.13 \\
\hline Sc16 & 3 & 0.62 & 2 & 0.48 & 3 & 0.25 & 2 & 0.05 & 4 & 0.35 \\
\hline Sc17 & 2 & 0.11 & 4 & 0.43 & 2 & 0.05 & 3 & 0.55 & 5 & 0.28 \\
\hline Sc18 & 3 & 0.68 & 3 & 0.54 & 3 & 0.21 & 4 & 0.58 & 5 & 0.50 \\
\hline Sc19 & 3 & 0.65 & 3 & 0.54 & 3 & 0.25 & 3 & 0.58 & 3 & 0.50 \\
\hline Sc20 & 2 & 0.11 & 2 & 0.39 & 2 & 0.11 & 2 & 0.49 & 3 & 0.27 \\
\hline Sc21 & 3 & 0.60 & 3 & 0.51 & 3 & 0.11 & 2 & 0.20 & 3 & 0.35 \\
\hline Sc22 & 4 & 0.75 & 4 & 0.56 & 4 & 0.21 & 7 & 0.70 & 9 & 0.55 \\
\hline Mean & 3.50 & 0.57 & 4.07 & 0.50 & 3.50 & 0.20 & 4.29 & 0.51 & 8.00 & 0.44 \\
\hline SD & 1.74 & 0.21 & 2.53 & 0.19 & 1.56 & 0.11 & 2.13 & 0.23 & 6.26 & 0.24 \\
\hline
\end{tabular}

